\title{
Simultaneous Phosphorus and Si Self-Diffusion in Extrinsic, Isotopically Controlled Silicon Heterostructures
}

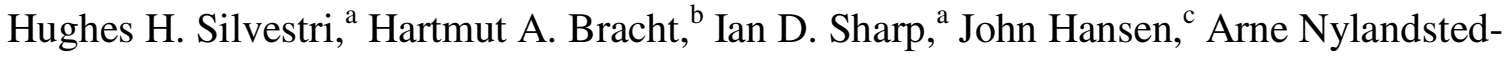 \\ Larsen, ${ }^{\mathrm{c}}$ and Eugene E. Haller ${ }^{\mathrm{a}}$ \\ ${ }^{a}$ Department of Materials Science and Engineering, University of California, Berkeley and \\ Materials Science Division, Lawrence Berkeley National Laboratory, Berkeley, CA 94720 \\ ${ }^{\mathrm{b}}$ Institut für Materialphysik, Universität Münster, Germany \\ ${ }^{c}$ Institute of Physics and Astronomy, University of Aarhus, Denmark
}

\begin{abstract}
We present experimental results of impurity and self-diffusion in an isotopically controlled silicon heterostructure extrinsically doped with phosphorus. As a consequence of extrinsic doping, the concentration of singly negatively charged native defects is enhanced and the role of these native defect charge states in the simultaneous phosphorus and Si self-diffusion can be determined. Multilayers of isotopically controlled ${ }^{28} \mathrm{Si}$ and natural silicon enable simultaneous analysis of ${ }^{30} \mathrm{Si}$ self-diffusion into the ${ }^{28} \mathrm{Si}$ enriched layers and phosphorus diffusion throughout the multilayer structure. An amorphous $260 \mathrm{~nm}$ thick Si cap layer was deposited on top of the Si isotope heterostructure. The phosphorus ions were implanted to a depth such that all the radiation damage resided inside this amorphous cap layer, preventing the generation of excess native defects and enabling the determination of the Si self-diffusion coefficient and the phosphorus diffusivity under equilibrium conditions. These samples were annealed at temperatures between 950 and $1100{ }^{\circ} \mathrm{C}$ to study the diffusion. Detailed analysis of the diffusion process was performed on the basis of a $\mathrm{P}$ diffusion model which involves neutral and positively charged mobile $\mathrm{P}$ species and neutral and singly negatively charged self-interstitial.
\end{abstract}

\section{INTRODUCTION}

While dopant and self-diffusion in silicon are known to be mediated by interstitial silicon atoms and/or lattice vacancies [1], the precise role of native defects in dopant and self-diffusion in Si remains an unresolved aspect of the current understanding of diffusion in Si. The use of stable isotope heterostructures has been shown to be a valuable technique for accurately determining the temperature dependence of the Si self-diffusion coefficient, described by a single activation enthalpy [2]. While this result demonstrated the value of isotope multilayers in studying self-diffusion, it alone was unable to yield information on the role of native defects on self-diffusion in Si. In the case that these native point defects are charged, their concentration is affected by the position of the Fermi level [3]. In particular, extrinsic n-type (p-type) doping favors negatively (positively) charged defects. Therefore the observation of extrinsic dopant diffusion along with self-diffusion will shed light on the impact of doping and dopant diffusion on the formation and diffusion of native point defects. This interrelation between self- and dopant diffusion can be studied by utilizing isotopically enriched $\mathrm{Si}$ isotope heterostructures for dopant diffusion experiments.

Previous experiments under extrinsic conditions on the simultaneous diffusion of the p-type dopant boron and Si generated information on the role of the singly positively charged selfinterstitial to self-diffusion and indicated boron diffusion in Si via a kick-out mechanism 
involving the neutral and singly positively charged self-interstitial [4,5]. The n-type dopant As was also studied via simultaneous dopant and self-diffusion in a Si isotope heterostructure under extrinsic conditions. These experiments provided information on the role of a singly negatively charged and a neutral native defect to self-diffusion, however the identity (interstitial or vacancy) of the negatively charged native defect was unresolved [5,6]. In order to clarify the nature of the singly negatively charged native defect in As diffusion and to determine the mechanisms of $\mathrm{P}$ diffusion, we studied the impact of the diffusion of the n-type dopant $\mathrm{P}$ on self-diffusion in a $\mathrm{Si}$ isotope heterostructure.

Intrinsic phosphorus diffusion was demonstrated to be retarded under nitridation (vacancy injection) conditions [7, 8]. From this retardation, it was concluded that the intrinsic $\mathrm{P}$ diffusion was entirely interstitially mediated [9]. For heavy P doping yielding extrinsic conditions, the retardation of $\mathrm{P}$ diffusion under nitridation was observed to decrease, signifying an increased contribution of vacancies to the diffusion [10]. Additionally, the P diffusivity was found to be proportional to the square of the $\mathrm{P}$ concentration for high $\mathrm{P}$ concentrations, indicating that the vacancy mechanism involving a doubly negatively charged vacancy $\left(\mathrm{V}^{-2}\right)$ is the dominant diffusion mechanism at high $\mathrm{P}$ concentrations [11]. This work will provide insight into the native defects involved in the diffusion of $\mathrm{P}$ in $\mathrm{Si}$ as well as contributions of vacancies and selfinterstitials to Si self-diffusion, but also yields a more detailed insight into the mechanism of dopant diffusion compared to previous studies that were restricted to the analysis of dopant profiles alone [12].

\section{EXPERIMENTAL}

The simultaneous dopant and self-diffusion was achieved by using a heterostructure consisting of alternating layers of enriched ${ }^{28} \mathrm{Si}$ and natural $\mathrm{Si}$, grown on a $\mathrm{Si}$ wafer of natural isotopic composition via ultra-high vacuum chemical vapor deposition at Lawrence Semiconductor Research Laboratories. Five ${ }^{28}$ Si enriched layers were grown separated by natural Si layers. Each individual layer was $120 \mathrm{~nm}$ thick. The dopant ions were introduced via implantation into a $250 \mathrm{~nm}$ thick amorphous Si cap layer. This cap was deposited via low temperature molecular beam epitaxy (LT-MBE) and was used to prevent the transient enhanced diffusion (TED) which results from the introduction of excess native defects from the implantation damage. Previous results on this structure demonstrated that ion implantation into the amorphous cap layer and even the re-crystallization of the amorphous layer during annealing does not cause any significant transient enhanced diffusion of Si [6].

To create the heavily doped phosphorus diffusion source, sequential implants of phosphorus were performed at $65 \mathrm{keV}$ with a dose of $7 \times 10^{15} \mathrm{~cm}^{-2}$ and $75 \mathrm{keV}$ at $7 \times 10^{15} \mathrm{~cm}^{-2}$ into the amorphous cap layer. After implantation, the samples were diced into $4 \times 4 \mathrm{~mm}^{2}$ pieces. Each individual sample was sealed in a silica ampoule under $0.5 \mathrm{~atm}$ Ar, to aid thermal transport and to prevent oxidation, and annealed in a resistance-heated tube furnace for appropriate times and temperatures. A type-S, Pt-Rh, thermocouple was used to monitor the temperature. The ampoules were quenched in water to terminate the diffusion. Depth profiles for ${ }^{30} \mathrm{Si}$ and ${ }^{31} \mathrm{P}$ were measured via Secondary Ion Mass Spectrometry (SIMS) on a Cameca $4 \mathrm{f}$ at Accurel Systems (Sunnyvale, CA). 


\section{RESULTS}

Phosphorus implanted samples were annealed at temperatures between 950 and $1100{ }^{\circ} \mathrm{C}$. From the SIMS depth profiles of Si and P, a model for simultaneous $\mathrm{P}$ and Si diffusion was generated based on the following reactions:

$$
\begin{aligned}
& P I^{o}\left(P_{i}^{o}\right) \Leftrightarrow P_{s}^{+}+I^{o}+e \\
& P^{o}\left(P_{i}^{o}\right) \Leftrightarrow P_{s}^{+}+I^{-} \\
& P I^{o}\left(P_{i}^{o}\right)+V^{o} \Leftrightarrow P_{s}^{+}+e \\
& P I^{+}\left(P_{i}^{+}\right) \Leftrightarrow P_{s}^{+}+I^{o} \\
& P I^{+}\left(P_{i}^{+}\right)+V^{o} \Leftrightarrow P_{s}^{+}
\end{aligned}
$$

where PI and $\mathrm{P}_{\mathrm{i}}$ represent the phosphorus-interstitial pair and interstitial phosphorus atom, respectively, for the given charge states. The vacancy and interstitial of the corresponding charge state are represented by V and I, while $e$ represents electrons used in the reaction for charge neutrality. The interstitial phosphorus representation is given in each equation along with PI because the model has only been generated for PI and therefore, strictly from a modeling standpoint, $\mathrm{P}_{\mathrm{i}}$ cannot be ruled out as a possible replacement for PI in the above equations without further modeling.

Figure 1 is a plot of the SIMS data for a sample annealed at $1100{ }^{\circ} \mathrm{C}$ for 30 minutes along with the best-fit simulation of the above reactions to the data set. From the figure it is evident that the model described by the reactions in Equations 1 to 5 accurately describe both $\mathrm{P}$ and $\mathrm{Si}$ diffusion simultaneously.

The reactions described by Equations 1, 2, and 4 represent either the interstitialcy or kickout mechanism, depending on whether the P defect is a PI pair (interstitialcy) or interstitial P atom (kick-out). To accurately describe the experimental profiles, the interstitial mediated reactions required the involvement of both neutral and singly negatively charged self-interstitials, $\mathrm{I}^{\mathrm{O}}$ and $\mathrm{I}^{-}$, along with neutral and singly positively charged mobile $\mathrm{P}$ species. The two vacancy

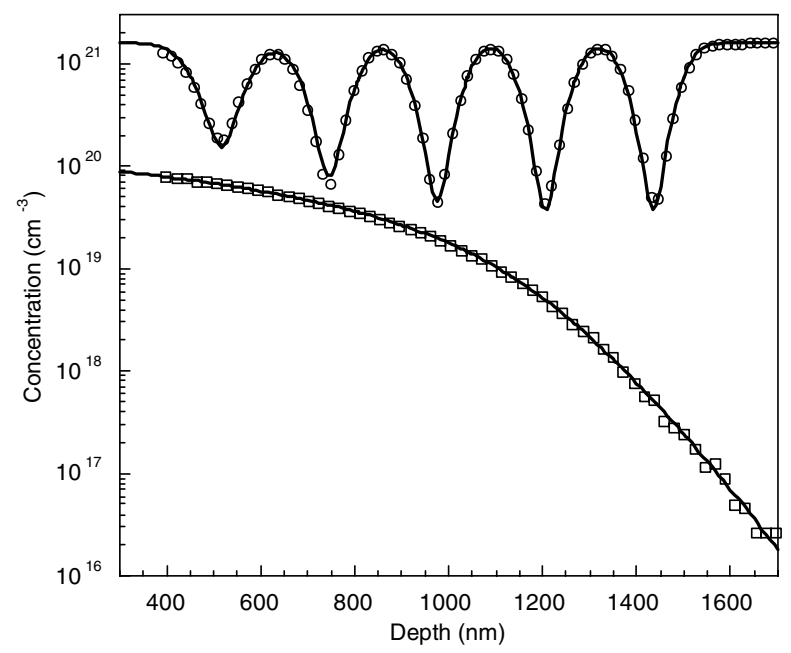

Figure 1 Plot of the SIMS depth profile of ${ }^{31} \mathrm{P}$ (squares) and ${ }^{30} \mathrm{Si}$ (circles) in a Si isotope structure annealed at $1100{ }^{\circ} \mathrm{C}$ for 30 minutes. Also shown in the figure is the best fit simulations (lines) generated from Equations 1 to 5. Every third data point is displayed for clarity. 
mediated reactions, 3 and 5, can either be represented as the pair assisted annihilation mechanism or the dissociative mechanism depending on the identity of the mobile P species which may be resolved by further modeling.

\section{DISCUSSION}

The model proposed here differs from that of the most widely accepted model of the reactions involved in $\mathrm{P}$ diffusion. The model proposed by Uematsu, [12], is based on a kick-out mechanism of interstitial diffusion involving a neutral interstitial, $\mathrm{I}^{\mathrm{O}}$, and a vacancy mechanism for vacancy mediated diffusion involving a singly and doubly negatively charged vacancy, $\mathrm{V}^{-}$ and $\mathrm{V}^{-2}$. In the model proposed in this work, no contribution from a vacancy mechanism is required and no evidence of the involvement of a doubly negatively charged native defect is observed, while a negatively charged interstitial is required along with a positively charged $\mathrm{P}$ species. It should be noted that, in contrast to the present work, the experimental data upon which Uematsu formed his model contained no Si self-diffusion profile. The model was based on fitting to a set of $\mathrm{P}$ diffusion profiles only. To check the accuracy of the Uematsu model for fitting both $\mathrm{Si}$ and $\mathrm{P}$ diffusion simultaneously, the model of Uematsu was applied to the same data set as shown in Figure1, a $\mathrm{Si}$ isotope heterostructure annealed at $1100{ }^{\circ} \mathrm{C}$ for 30 minutes. The results of the fit of the Uematsu model to the simultaneous Si and P diffusion from this work are presented in Figure 2 along with the reaction equations of the Uematsu model. The dashed line in Figure 2 represents the best-fit of the $\mathrm{P}$ data to the experimental results. It is clear that under the model proposed by Uematsu, the $\mathrm{P}$ diffusion profiles generated in this work can be accurately described. However, the simultaneous modeling of the Si self-diffusion profile along with the best-fit of the P using the Uematsu model does not yield an accurate description of the $\mathrm{Si}$ self-diffusion. The solid line in Figure 2 represents an attempt to generate a best-fit of the $\mathrm{Si}$ profile from the simultaneous $\mathrm{Si}$ and $\mathrm{P}$ data using the Uematsu model. An accurate representation of the Si profile can be generated, but the $\mathrm{P}$ profile generated by that fit is no longer appropriate. Therefore it appears that the model proposed by Uematsu, while accurate for the description of $\mathrm{P}$ diffusion, cannot describe the simultaneous diffusion of $\mathrm{Si}$ and $\mathrm{P}$.

From the fitting of the experimental data to the model given in Equations 1 to 5, diffusion coefficients for Si diffusion via interstitials and $\mathrm{P}$ diffusion over the temperature range of $950{ }^{\circ} \mathrm{C}$ to $1100{ }^{\circ} \mathrm{C}$ were generated. The temperature dependence of these values is plotted as an Arrhenius relation in Figure 3. Also plotted in Figure 3 are lines representing the previous results for intrinsic $\mathrm{P}$ diffusion [13] and the results for Si diffusion via a negatively charged native defect, from the simultaneous As and Si diffusion [5,6]. The temperature dependence of the data was derived from exponential fits to the diffusivity values. Close agreement is observed between the literature values for P diffusion in Si [13] and the present work. The agreement of the diffusion coefficient for the negatively charged native defect between the As and $\mathrm{P}$ results is considered to be a check of consistency of the technique of simultaneous dopant and selfdiffusion in $\mathrm{Si}$ isotope heterostructures.

The temperature dependences of the P diffusion coefficient and the $\mathrm{I}^{-}$contribution to selfdiffusion are given by the following expressions (both results refer to electronically intrinsic conditions):

$$
D_{P}=0.36_{-0.23}^{+0.64} \cdot \exp \left(-\frac{(3.33 \pm 0.11) \mathrm{eV}}{k T}\right) \mathrm{cm}^{2} \mathrm{~s}^{-1}
$$




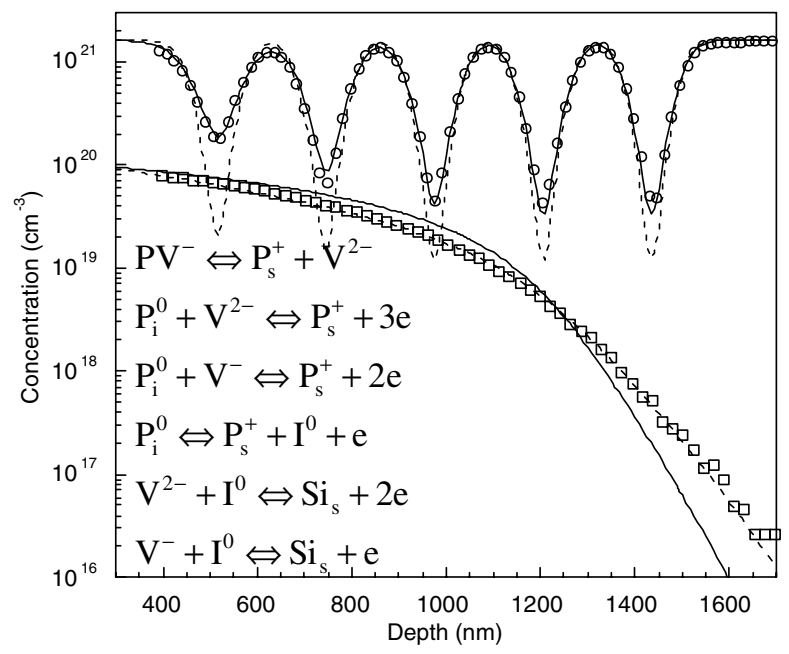

Figure 2 SIMS concentration profiles of ${ }^{30} \mathrm{Si}$ (circles) and ${ }^{31} \mathrm{P}$ (squares) for a sample annealed at $1100{ }^{\circ} \mathrm{C}$ for 30 minutes, along with simulations for the model proposed by Uematsu [12]. The dashed line represents best fit of Uematsu's model to the P profile, while the solid line is the best fit of Uematsu's model to the Si profile. Only every third SIMS data point is shown for clarity. The diffusion reactions proposed by Uematsu are inset [12].

$$
D_{I^{-}}^{S D}=\left(4.3_{-3.8}^{+32.0}\right) \cdot 10^{4} \cdot \exp \left(-\frac{(5.40 \pm 0.24) e V}{k T}\right) c^{2} s^{-1}
$$

where $\mathrm{k}$ is Boltzmann's constant and $\mathrm{T}$ is the temperature in Kelvin. The values for the activation energy, $\mathrm{Q}$, from these expressions confirm the agreement with previous results for $\mathrm{P}$ $(\mathrm{Q}=3.69 \mathrm{eV}[13])$ and the negatively charged defect contribution from the previous As and $\mathrm{Si}$ diffusion experiments $(\mathrm{Q}=5.18 \mathrm{eV}[5,6])$.

\section{CONCLUSIONS}

The proposed model has several implications for the mechanisms and properties of $\mathrm{P}$ diffusion in Si. First, it indicates that interstitials are more important than vacancies for $\mathrm{P}$ diffusion below the $\mathrm{P}$ concentration of $2 \times 10^{20} \mathrm{~cm}^{-3}$, which was the highest concentration observed in this study. Secondly, this model requires the existence of a singly negatively charged Si self-interstitial defect and a singly positively charged P mobile species. No doubly negatively charged defect or a vacancy mechanism was needed for accurate fitting of the profiles. Finally, it should be noted that the tail in the P diffusion SIMS profile, seen in Figures 1 and 2, could not be modeled solely by the interstitial supersaturation generated from $\mathrm{P}$ diffusion, as in the case of $\mathrm{B}$ diffusion [4]. For the $\mathrm{P}$ diffusion profiles, the extended tail could only be fit by the additional incorporation of the positively charged mobile $\mathrm{P}$ species, which would lead to a more extended tail than a neutral $\mathrm{P}$ defect, as a result of charge repulsion from the ionized substitutional $\mathrm{P}$ species. It is this result that leads to the conclusion of the existence of a positively charged mobile $\mathrm{P}$ species. 


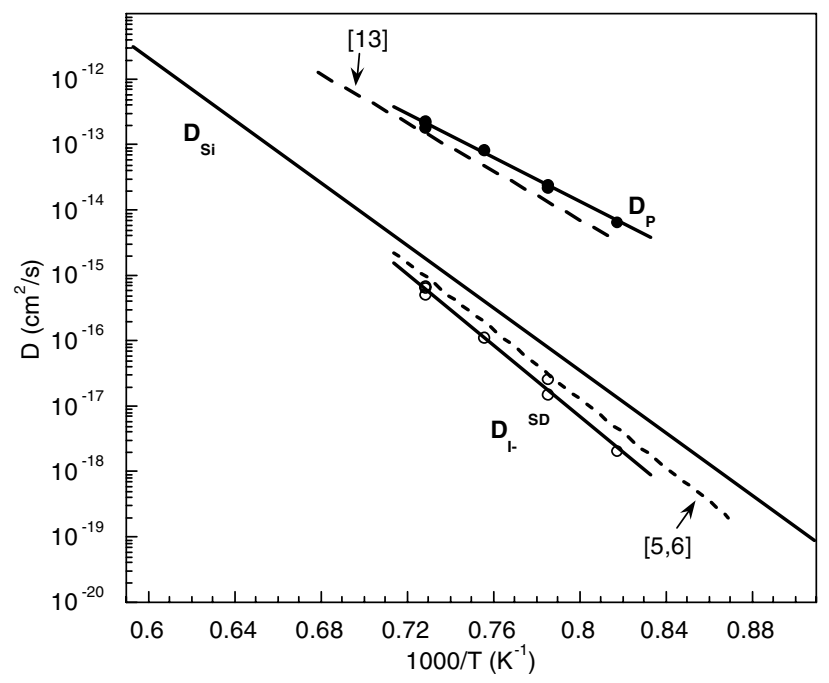

Figure 3 Plot of the diffusion coefficient for P and Si as a function of inverse absolute temperature. The contribution of the negatively charged self-interstitial derived from simultaneous Si and P diffusion (open circles, solid line) is shown in close agreement with the results for simultaneous As and Si diffusion (short dashed line) [5,6]. The values for the intrinsic $\mathrm{P}$ diffusion (close circles, solid line) are plotted along with the values from literature [13] (long dashed line). The Arrhenius dependence of the total Si self-diffusion, $\mathrm{D}_{\mathrm{Si}}$ [2], is also shown.

\section{ACKNOWLEDGEMENTS}

This work was supported in part by a UC Discovery Grant from the Industry-University Cooperative Research Program (IUCRP), by US NSF Grant No. DMR-0109844, and by the Director, Office of Science, Office of Basic Energy Sciences, Division of Materials Sciences and Engineering, of the U.S. Department of Energy under Contract No. DE-AC03-76SF00098.

\section{REFERENCES}

1. P.M. Fahey, P.B. Griffin, and J.D. Plummer, Reviews of Modern Physics, 61289 (1989).

2. H. Bracht, E.E. Haller, and R. Clarke-Phelps, Phys. Rev. Lett. 81393 (1998).

3. W. Shockley and J.L. Moll, Phys. Rev. 1191480 (1960).

4. I.D. Sharp, H.A. Bracht, H.H. Silvestri, S.P. Nicols, J.W. Beeman, J. Hansen, A. Nylandsted Larsen, E.E. Haller, Mat. Res. Soc. Symp. Proc. 719 F13.11.1 (2002).

5. H. Bracht, H.H. Silvestri, I.D. Sharp, S.P. Nicols, J.W. Beeman, J.L. Hansen, A. Nylandsted Larsen, E.E. Haller, Institute of Physics Conference Series 171 C3.8 (2003).

6. H.H. Silvestri, I.D. Sharp, H.A. Bracht, S.P. Nicols, J.W. Beeman, J. Hansen, A. Nylandsted Larsen, E.E. Haller, Mat. Res. Soc. Symp. Proc. 719 F13.10.1 (2002).

7. S. Mizuo, T. Kusaka, A. Shintani, M. Nanba, and H. Higuchi, J. Appl. Phys. 543860 (1983).

8. P. Fahey, G. Barbuscia, M. Moslehi, and R.W. Dutton, Appl. Phys. Lett. 46784 (1985).

9. T.Y. Tan, and U. Gösele, Appl. Phys. A 371 (1985).

10. P. Fahey, Ph.D. thesis, Stanford University (1985).

11. R.B. Fair, and J.C.C. Tsai, J. Electrochem. Soc. 1241107 (1977).

12. M. Uematsu, J. Appl. Phys. 822228 (1997).

13. J.S. Makris, and B.J. Masters, J. Electrochem. Soc. 1201252 (1973). 